\title{
Toxin-based models to investigate demyelination and remyelination
}

Authors: Christopher E McMurran, Chao Zhao, Robin J M Franklin

\section{Summary}

Clinical myelin diseases, and our best experimental approximations, are complex entities in which demyelination and remyelination proceed unpredictably and concurrently. These features can make it difficult to identify mechanistic details. Toxin-based models offer lesions with predictable spatiotemporal patterns and relatively discrete phases of damage and repair: a simpler system to study the relevant biology and how this can be manipulated. Here, we discuss the most widely used toxin-based models, with a focus on lysolecithin, ethidium bromide and cuprizone. This includes an overview of their respective mechanisms, strengths and limitations and step-by-step protocols for their use.

\section{Key Words}

"demyelination, remyelination, animal models, toxin, lysolecithin, lysophosphatidylcholine, ethidium bromide, cuprizone"

\section{Introduction}

The causes of demyelinating disease are many, and the consequences can be devastating for a patient. Several toxins have been reported to cause clinical demyelination in humans, including sodium cyanate and the antiseptic hexachlorophene [1]. However, these cases are rare when compared to the autoimmune diseases multiple sclerosis and neuromyelitis optica, or even genetic mutations as occur in Pelizaeus-Merzbacher disease or adrenoleukodystrophy. Toxin-based models do not attempt to replicate the complex pathogenesis of these diseases, but rather provide a reductionist setting to study certain aspects, where kinetics are predictable and confounding variables minimised. This approach has yielded substantial insight into the biology of demyelination and remyelination, with much relevance to human disease.

\subsection{Types of toxin-based model}

Due to the diversity in the range of toxins used to cause demyelination and their modes of delivery, this chapter will focus on three of the most common models. Focal injection of two substances (lysolecithin and ethidium bromide) will be discussed, as will administration of cuprizone in the diet. Other toxins reported to cause primary demyelination in animal models include 6-aminonicotinamide [2], antibodies to myelin proteins (e.g. [3]) and bacterial endotoxin [4], and these are briefly summarised in Table 1. 
Table 1: Overview of toxin-based models for demyelination/remyelination.

\begin{tabular}{|c|c|c|c|}
\hline Toxin & $\begin{array}{c}\text { Focal / } \\
\text { Systemic }\end{array}$ & Mechanism & Principal uses \\
\hline Lysolecithin & Focal & $\begin{array}{ll}\text { - } & \text { Detergent effect on } \\
\text { oligodendrocytes } \\
\text { - } & \text { Activates immune cells }\end{array}$ & $\begin{array}{l}\text { - Most commonly used focal } \\
\text { model, with very well-defined } \\
\text { kinetics } \\
\text { - Electrophysiological recordings } \\
\text { in a specific location }\end{array}$ \\
\hline Ethidium Bromide & Focal & $\begin{array}{ll}\text { - } & \text { Intercalates in DNA } \\
\text { - } & \text { Affects all nuclei in } \\
& \text { lesion, sparing axons }\end{array}$ & $\begin{array}{l}\text { - Remyelination in the relative } \\
\text { absence of astrocytes } \\
\text { - Lesions in CCP can be combined } \\
\text { with an in-dwelling catheter for } \\
\text { infusion }\end{array}$ \\
\hline Cuprizone & Systemic & $\begin{array}{ll}\text { Disturbs copper } \\
\text { homeostasis, causing } \\
\text { metabolic stress } \\
\text { - } \\
\text { Oligodendrocytes are } \\
\text { especially vulnerable } \\
\text { and undergo apoptosis }\end{array}$ & $\begin{array}{l}\text { - No surgical procedures } \\
\text { required } \\
\text { - Remyelination with intact } \\
\text { blood-brain barrier [5] } \\
\text { - Similarities with pattern III } \\
\text { lesions in multiple sclerosis [6] }\end{array}$ \\
\hline 6-aminonicotinamide & $\begin{array}{l}\text { Focal, with } \\
\text { systemic } \\
\text { effects on } \\
\text { grey matter }\end{array}$ & \begin{tabular}{|l} 
\\
Bikely to involve vitamin \\
antagotinic acid) \\
gliotoxicity [7]
\end{tabular} & $\begin{array}{l}\text { - Remyelination in the relative } \\
\text { absence of astrocytes } \\
\text { (Largely superseded by other } \\
\text { models) }\end{array}$ \\
\hline $\begin{array}{l}\text { Anti-galactocerebroside } \\
\text { + complement }\end{array}$ & Focal & $\begin{array}{l}\text { Antibody against a major } \\
\text { constituent of myelin } \\
\text { causes selective fixation } \\
\text { of complement }\end{array}$ & - Highly axon-sparing lesion \\
\hline $\begin{array}{l}\text { Bacterial endotoxin } \\
\text { (lipopolysaccharide) }\end{array}$ & Focal & $\begin{array}{l}\text { Direct Toll-Like Receptor } \\
\text { signalling to } \\
\text { oligodendrocytes and } \\
\text { astrocytes [8] } \\
\text { - } \\
\text { Activates immune cells }\end{array}$ & $\begin{array}{l}\text { - Focal model of immune- } \\
\text { mediated demyelination } \\
\text { - Similarities with pattern III } \\
\text { lesions in multiple sclerosis [4] }\end{array}$ \\
\hline
\end{tabular}

\subsubsection{Lysolecithin}

Lysolecithins (lysophosphatidylcholines, LPCs) are hydrolysis products of phospholipids, which are found naturally in cell membranes and have physiological roles in mediating the phagocytosis of apoptotic cells [9]. The demyelination literature typically refers to the singular lysolecithin (lysophosphatidylcholine), which is in fact a mixture of lysolecithins with different fatty acid tails, prepared by the enzymatic action of phospholipase $\mathrm{A}_{2}$ on a natural substrate rich in phospholipids such as egg yolk (for example: Sigma L4129). Focal injection of lysolecithin was first shown to cause CNS demyelination in the mouse spinal cord in the 1970s [10] and subsequent studies have shown similar lesions in other species including rat [11] and rabbit [12], and other white matter tracts such as the corpus callosum [13] and caudal cerebellar peduncle (CCP)[14].

Lysolecithin selectively destroys myelin, and kills the majority of oligodendrocytes [15] in an ellipsoid region spanning several millimetres. Following injection, there is marked inflammation in the core of the lesion with astrogliosis at the border [16]. Axonal injury can be more pronounced than in other toxin-based models. Remyelination occurs extensively and rapidly in 
young animals (within one month in the young adult mouse [16]) and is mainly carried out by new oligodendrocytes, generated from oligodendrocyte progenitor cells (OPCs)[17]. Remyelination by Schwann cells can also occur in the centre of a lesion, particularly if it is large or there is excessive astrocyte loss. In older animals the regeneration of myelin sheaths occurs more slowly [18].

The demyelinating action of lysolecithin is likely a combination of two effects (Fig 1a). Firstly, being made up of amphipathic molecules, concentrated lysolecithin can act as a detergent, compromising the integrity of cell membranes [19]. Oligodendrocytes are particularly vulnerable to this effect, which may be in part because other cell types are able to metabolise lysolecithin, limiting its concentration in their membranes [20]. Secondly, lysolecithin can signal directly to immune cells, being a chemoattractant signal for monocytes and lymphocytes $[21,22]$ and direct activator of monocytes, macrophages, microglia and lymphocytes [23-25]. Thus the toxin may directly activate immune cells to strip axons of their myelin, which is already compromised by the detergent action. Evidence for an immune-mediated component is that lysolecithin-induced demyelination is reduced when immune signalling molecules are neutralised [26], or in mice devoid of T cells [27]. Despite these roles in the pathology, macrophages, microglia and $\mathrm{T}$ cells are also necessary for efficient remyelination [28-30]. Demyelination does not depend on CXCR2+ neutrophils, which are essential in the cuprizone model [31].

By virtue of its relatively high selectivity to oligodendrocytes, lysolecithin can also been used to study demyelination and remyelination in organotypic slice culture. Slices of cerebellum several hundred micrometres thick are most commonly used, as their largely self-contained neural networks allows good survival of myelinated Purkinje cells ex vivo [32]. This model has been successful in both rat [32] and mouse tissue [33] and has proved useful for screening compounds suspected to influence remyelination in a shorter timescale than for live animals [33-35].

\subsubsection{Ethidium bromide}

Ethidium bromide (EB) is a planar molecule that can intercalate between base pairs in double-stranded DNA [36] to inhibit transcription and replication. This property has made it useful as a veterinary cytotoxic drug to treat trypanosomiasis [37], as well as for visualisation of nucleic acids for research purposes. Focal demyelination by EB injection into a white matter tract was first demonstrated in the spinal cords of cats in the 1980s [38] and has since become well established in mice and rats, with other locations including the CCP white matter tract [14] and the grey matter of the hippocampus [39].

Injected EB is cytotoxic to any cell with a cell body in the lesion area (Fig 1b). This will include oligodendrocytes and astrocytes [38] as well as OPCs [40], but axons projecting from distant nuclei are spared, providing especially high doses are not used. This means that EB and lysolecithin lesions differ in some crucial respects. EB lesions tend to be larger and remyelination occurs more slowly, likely due to the death of progenitor cells and astrocytes/microglia, which must 
be recruited from surrounding intact tissue [14]. The death of astrocytes also results in an increased contribution of Schwann cells to remyelination, as astrocytes inhibit OPCs from adopting a Schwann cell fate [41, 42]. Clearance of myelin debris is frequently delayed in EB lesions, contributing to a slower rate of remyelination $[43,44]$, a probable consequence of microglial death and an impaired inflammatory response. Thus comparisons between EB and lysolecithin lesions have helped to disentangle some of the important roles that astrocytes and microglia play in remyelination.

\subsubsection{Cuprizone}

In contrast to the focal injection of lysolecithin or ethidium bromide, the copper chelator cuprizone (biscyclohexanone oxalyldihyrazone) is administered to animals in their diet. This abrogates the need for surgical equipment and expertise, making it the toxin-based model of choice for many groups studying remyelination. Since the 1960s, cuprizone has been known to cause demyelination in mice [45] though subsequent studies using a range of species, strains, ages and doses showed much variability in the degree of demyelination. To achieve consistency and reproducibility, most experimenters feed a $0.2 \%$ cuprizone diet to young adult male C57BL/6 mice for a period of 4-6 weeks, which has been shown to give a predictable pattern of demyelination with the scope for transgenic studies [46].

Demyelination occurs most notably in the corpus callosum, but also at other sites including the hippocampus, external capsule, cerebellar peduncles and cerebral cortex [47]. Callosal demyelination becomes gradually apparent from 3 weeks of treatment, and is accompanied by extensive inflammation and astrogliosis [46]. The corpus callosum is most affected towards its caudal end (Fig 2), thus it is important that equivalent locations are selected for meaningful comparison [47]. Remyelination overlaps with continued demyelination: at 3 weeks OPCs begin to accumulate [48] and mRNA for myelin proteins is detected from week 5 [49]. Often the mice are returned to their regular diet for 1-2 weeks to allow a period of remyelination in the absence of active demyelination. Remyelination is substantial after a 3 weeks recovery, with restoration of the perinodal architecture [50], although this may not entail complete functional regeneration as axonal degeneration and locomotor impairment can reappear several months later [51].

Cuprizone has a high affinity for copper, an important cofactor for metabolic reactions including oxidative phosphorylation in mitochondria. However, the exact mechanism by which it causes demyelination remains a matter of controversy [52]. One hypothesis suggests that the toxin itself remains in the gut, where it chelates copper and brings about a systemic deficiency and failure of copper-dependent processes $[53,54]$. An alternative theory proposes that cuprizone can distribute into the plasma and CNS, where it locally disrupts the homeostasis of copper and other metals $[55,56]$. In any case, dietary cuprizone treatment leads to disturbances in cellular metabolism and selective apoptosis of oligodendrocytes, which are particularly vulnerable due to their high metabolic rate and low levels of antioxidants (Fig 1c) $[56,57]$. OPCs and astrocytes are 
largely spared, the latter of which are also thought to protect neurons through metabolic coupling [52]. However, it is important to note that cuprizone is a systemic toxin and will likely have off-target effects in the CNS as well as other tissues - notably the liver [58].

An initial phase of early oligodendrocyte death [59] is exacerbated by a second hit from the immune system. Full demyelination depends on the actions of neutrophils [31] and microglia [60] but not lymphocytes [61]. However, as with lysolecithin and EB lesions, the innate immune system also plays a vital role in remyelination, by clearing debris and secreting growth factors [30, 62, 63].

Aspects of the cuprizone paradigm can be modified to suit the specific needs of an experiment. A common version is the "chronic" cuprizone model, whereby mice receive a 12-week course of cuprizone and show impaired remyelination with more axonal damage $[64,65]$. In another variation, mice receive the mTOR inhibitor rapamycin, which blocks OPC differentiation and delays remyelination until the end of cuprizone treatment [66]. This allows separation of the demyelination and remyelination phases, as seen in lysolecithin or EB lesions, though the effect of rapamycin on the microglial and astrocyte responses remains to be fully characterised.

\subsection{Why use toxin-based models to study demyelination and remyelination?}

Toxin-based models are just one strategy to model myelin diseases in vivo, and should therefore be considered alongside other approaches. Alternatives include autoimmune models such as Experimental Autoimmune Encephalomyelitis (EAE) $[67,68]$, and infectious models such as Theiler's Murine Encephalomyelitis (TMEV) [69, 70], which are generally better representations of the effector aspects of multiple sclerosis. Genetic models have also furthered our understanding, for example the effects of mutations that cause myelin disease can be modelled in transgenic mice [71].

A key strength of toxin-based models is their predictability, both in their temporal and spatial patterns. Lysolecithin and EB lesions demonstrate a consistent sequence of events with daily resolution, giving a clear background on which to study factors that may influence the rate of demyelination or remyelination. Cuprizone also has a predictable course, albeit over a longer timescale. This is in contrast to EAE, where the sites of demyelination will vary between animals, and lesions of different ages will occur in the same animal, complicating interpretation. Focal lesions of specific white matter tracts can also be favourable for animal welfare, with phenotypes generally less severe than in EAE [72].

A separation between the demyelination and remyelination phases is another feature of the lysolecithin and EB models. Whilst this does not recapitulate the natural history of diseases such as multiple sclerosis, it has allowed pathways involved in pathology and regeneration to be disentangled. A notable example was identifying the positive contribution of the innate immune system to 
remyelination [28], which is obscured in other models by its roles in myelin damage.

Toxin-based models have generally been viewed as reductionist tools, whilst EAE and viral models are used as our best approximations to human disease. However, there is much heterogeneity between lesions in multiple sclerosis and some may in fact be better modelled using toxin-based approaches. For example cuprizone is thought to be one of the best experimental models for pattern III clinical demyelination [6]. It is clear that a continued synthesis of results from different approaches is necessary to advance the field of myelin research. Toxinbased models can be a valuable resource, so long as the strengths and limitations are considered and the choice of model is well suited to answer the question at hand. 


\section{Materials}

Protocols for a typical paradigm using each of our three toxins will be described:

- lysolecithin - focal injection into mouse ventral spinal cord

- ethidium bromide - focal injection into rat caudal cerebellar peduncle

- cuprizone - dietary administration

\subsection{Focal injection models}

Lysolecithin and ethidium bromide are both focally injected under anaesthesia and much of the equipment required is common to both.

\section{Materials required for lysolecithin spinal cord lesions:}

- $1 \%$ lysolecithin (see below) - at least $1 \mu \mathrm{l}$ per lesion

- Sterile gown and gloves

- Anaesthetic machine (isoflurane in oxygen)

- An injectable anaesthetic may be used if an inhalation set-up is not available.

- Operating table with a stage for mouse and two surgical stands

- 3-way manipulator for Hamilton syringe

- Operating microscope

- Heat pad and rectal probe to monitor temperature

- Clippers

- Disposable drapes

- Gauze swabs

- Viscotears (Alcon)

- Antiseptic solution e.g. Betadine ${ }^{\circledR}$

- Sterile saline

- Analgaesic e.g. buprenorphine, $0.05 \mathrm{mg} / \mathrm{kg}$ (Vetergesic (Champion Alstoe) contains $0.3 \mathrm{mg} / \mathrm{ml}$ buprenorphine, so can be diluted 1 in 6 in saline and used $100 \mu \mathrm{l} / 100 \mathrm{~g}$ )

- Cotton wool or similar (for bleeding)

- Instruments:

- Hamilton syringe with glass tip for injecting lysolecithin

- 30G dental needle to cut dura

- $5 \mathrm{ml}$ syringe and needle to administer saline

- $1 \mathrm{ml}$ syringe and needle to administer analgaesic

- Scalpel with small blade (e.g. No. 15)

- Fine-tipped tweezers (2 sets)

- Mosquito forceps

- Needle holder

- Scissors

- 6-0 sutures

- Recovery unit

Lysolecithin should be prepared in advance. Add $10 \mathrm{ml}$ sterile saline to $100 \mathrm{mg}$ lysolecithin (Sigma L4129), dissolve, then make aliquots to freeze at $-20^{\circ} \mathrm{C}$. On the day of lesioning, defrost aliquot and sonicate in a sonicating water bath for 20-30 minutes until clear. 


\section{Materials required for ethidium bromide CCP lesions:}

- $0.01 \%$ ethidium bromide (see below) - at least $4 \mu \mathrm{l}$ per lesion

- Sterile gown and gloves

- Anaesthetic machine (isoflurane in oxygen)

- An injectable anaesthetic may be used if an inhalation set-up is not available.

- Operating table with a small animal stereotactic frame

- Operating microscope

- Heat pad and rectal probe to monitor temperature

- Clippers

- Disposable drapes

- Gauze swabs

- Viscotears (Alcon)

- Antiseptic solution e.g. Betadine ${ }^{\circledR}$

- Sterile saline

- Analgaesic e.g. buprenorphine, $0.05 \mathrm{mg} / \mathrm{kg}$ (Vetergesic (Champion Alstoe) contains $0.3 \mathrm{mg} / \mathrm{ml}$ buprenorphine, so can be diluted 1 in 6 in saline and used $100 \mu \mathrm{l} / 100 \mathrm{~g}$ )

- Cotton wool or similar (for bleeding)

- Instruments:

- $10 \mathrm{ml}$ Hamilton syringe with $26 \mathrm{G}$ cone tip needle for injecting EB

$\circ 5 \mathrm{ml}$ syringe and needle to administer saline

- $1 \mathrm{ml}$ syringe and needle to administer analgaesic

- Scalpel with small blade (e.g. No. 15)

○ Surgical drill

- Retractor

- Needle holder

- Scissors

- 5-0 sutures

- Recovery unit

Ethidium bromide can be purchased as a liquid and diluted in PBS to $0.01 \%$.

\subsection{Dietary cuprizone model}

\section{Materials required for the cuprizone model:}

- cuprizone diet (see below)

- biological safety cabinet to open cages

- scales for weighing mice

The easiest option is to purchase a diet with cuprizone incorporated at $0.2 \%$. A number of suppliers are able to provide this, including Envigo (TD.140804) and SDS (as a "Custom Made Diet"). A common alternative is to buy cuprizone separately and mix it with powdered chow. The diet should not be autoclaved, as cuprizone is heat-sensitive, and it should be stored in the dark and refrigerated. 


\section{Methods}

Surgical procedures should be carried out wearing a sterile gown and gloves, with good aseptic technique.

\subsection{PROTOCOL: Lysolecithin lesion in mouse ventral spinal cord}

Spinal cord lesions are normally carried out on mice aged 8 weeks and onwards. The procedure should take less than 30 minutes and sterile saline can be applied periodically to prevent the wound from drying out.

1. Prepare anaesthetic machine, operating microscope, operating table with stage suitable for mice, and sterile surgical instruments.

2. Prepare the following syringes:

- Glass-tipped Hamilton syringe with 1\% lysolecithin - ensure there are no bubbles and attach this to a 3-way manipulator fitted to a stand

- $1 \mathrm{ml}$ syringe of analgaesic e.g. buprenorphine, $0.05 \mathrm{mg} / \mathrm{kg}$.

3. Weigh mouse and transfer to anaesthetic chamber to begin induction of anaesthesia with isoflurane/oxygen. When effective, transfer mouse to operating stage equipped with mask for maintaining anaesthesia throughout the procedure. Position mouse prone with head away from operator.

4. Inject analgaesic subcutaneously. Position the temperature probe and apply 1 drop of Viscotears liquid gel to each eye.

5. Prepare skin by shaving the back around the natural "hump" and clean the shaved area with antiseptic solution. Do not treat excessively to prevent heat loss. Cut a small access window in a sterile drape and lay over mouse.

6. Begin operating after verifying the mouse has no response to pain. Start with a $0.5 \mathrm{~cm}$ midline incision at the top of the hump on the back (thoraco-lumbar region). Cut away the subcutaneous tissue to expose the muscles. Use a small scalpel blade to gently separate the muscles on both sides.

7. Under the microscope, find the space between two vertebrae using two pairs of fine-tipped tweezers and remove the soft tissues: first muscle and then the yellow ligament to expose the surface of the spinal cord. This should appear pale with the central vein visible in the midline. Care must be taken not to damage the spinal cord at this stage.

8. Use a pair of curved mosquito forceps to clamp the soft tissue above the exposed intervertebral space, then fix to a stand to minimise movement of the injection site from breathing. Use a very thin bevelled needle (such as a $30 \mathrm{G}$ dental needle) to open the dura just to the right of the central vein (Fig 3). 
9. Position the manipulator with the Hamilton syringe at a $70^{\circ}$ angle relative to the horizontal plane. Gentle adjust the tip of the syringe to touch the surface of the cord through the cut dura. Slowly lower the needle through the cord, until a slight deflection indicates contact with the vertebral bone underneath. Retract the needle fractionally so the deflected tip becomes straight.

10. Inject $0.5 \mu \mathrm{l}$ of toxin in about 20 seconds, then retract the needle about 0.2-0.25 $\mathrm{mm}$ and inject another $0.5 \mu \mathrm{l}$. Keep the needle in place for 1 minute.

11. Slowly raise the needle out of the spinal cord. And move away clamp and manipulator. Using a 6-0 suture, make one stitch to close the muscular layer, then 2-3 stitches for the skin. Lidocaine cream can be applied to reduce pain at the wound site.

12. Transfer the mouse to a warm chamber for recovery before returning it to the cage.

After the procedure, there should be no signs of distress or clinical abnormality. No special care is required and individual cages are not necessary. Occasionally a mouse may develop a unilateral hind leg impairment, which should recover in within 48 hours and will not prevent animal accessing food or water. External sutures are often removed by the mouse, and may need to be replaced if this occurs before wound closure.

\subsection{PROTOCOL: Ethidium bromide lesion in rat caudal cerebellar peduncle} Lesions of the CCP are typically carried out on rats aged 8 weeks and onwards. The procedure is more complex than spinal cord lesions due to the need to drill through the skull and use precise stereotactic coordinates. In total, it should take around 45-60 minutes.

1. Prepare anaesthetic machine, operating microscope, operating table with stereotactic frame, and sterile surgical instruments.

2. Prepare the following syringes:

- Hamilton syringe (with 26 G needle) with $0.01 \%$ ethidium bromide ensure there are no bubbles and attach this to stereotactic frame

- $5 \mathrm{ml}$ syringe of sterile saline - place in recovery unit to warm

- $1 \mathrm{ml}$ syringe of analgaesic e.g. buprenorphine, $0.05 \mathrm{mg} / \mathrm{kg}$.

3. Weigh rat and transfer to anaesthetic chamber to begin induction of anaesthesia with isoflurane/oxygen. When effective, inject analgaesic (subcutaneous) and 2.5-3 ml saline (intraperitoneal). Position the temperature probe.

4. Shave top of head from between the eyes to behind the ears. Scrub area with two gauze swabs wetted with antiseptic solution and apply 1 drop of 
Viscotears liquid gel to each eye.

5. Transfer rat to stereotactic frame equipped with mask for maintaining anaesthesia throughout the procedure. Open the mouth and pull tongue outside if possible. Attach the ear bars carefully, holding the animal. Each bar should be in the same position and an eye-closing reflex can be seen when the bar is correctly located. The head of the animal should be positioned flat, which can be verified once the skull is exposed.

6. Apply skin prep to the shaved area skin. Cut a small access window in a sterile drape and lay over rat.

7. Using a scalpel, make a $1.5 \mathrm{~cm}$ midline incision at the back of the head. Remove the connective tissue with scissors and forceps. Cotton wool can be used to stop the bleeding. Scrape away tissue overlying the bone with the scalpel.

8. With the aid of the microscope, locate the bregma and note its coordinates, $\beta$ (Fig 4). Touching the bones to move them slightly can aid with this.

9. Locate the lambda, which is defined as the midpoint of the curve of best fit along the lambda suture (Fig 4) rather than the intersection of the lambdoid and sagittal sutures [73]. Note its coordinates, $\lambda$.

10. Confirm that $\beta$ and $\lambda$ lie on the same horizontal plane, and adjust the position of the rat as necessary.

11. Calculate the stereotactic coordinates for the CCP using Table 2. These will depend on the size of the rat, and thus the $\beta-\lambda$ distance.

Table 2: Guide for calculating the coordinates of CCP relative to lambda, $\lambda$

\begin{tabular}{|c|c|c|c|c|c|c|c|}
\cline { 2 - 8 } \multicolumn{1}{c|}{} & B- $\boldsymbol{\lambda}(\mathbf{m m})$ & $<\mathbf{8 . 0}$ & $\mathbf{8 . 0} \mathbf{- 8 . 2}$ & $\mathbf{8 . 2} \mathbf{- 8 . 5}$ & $\mathbf{8 . 5}-\mathbf{9 . 0}$ & $\mathbf{9 . 0}-\mathbf{9 . 5}$ & $>\mathbf{9 . 5}$ \\
\hline \multirow{3}{*}{$\begin{array}{c}\text { Distance to } \\
\text { CCP (mm) }\end{array}$} & Anterior & 2.1 & 2.2 & 2.2 & 2.2 & 2.3 & 2.4 \\
\cline { 2 - 8 } & Ventral & $7.1-7.2$ & 7.2 & 7.2 & 7.2 & 7.3 & 7.4 \\
\cline { 2 - 8 } & Lateral & 2.5 & 2.5 & 2.5 & 2.5 & 2.6 & 2.65 \\
\hline
\end{tabular}

12. Apply the retractor. Position tip of drill at the point of entry.

13. Drill through the skull, advancing slowly. The syringe can be used periodically to confirm the hole is in the correct location.

14. When drilling is complete, move the syringe to the calculated coordinates for the CCP. The coordinates can be adjusted slightly if the needle catches on the edge of the burr hole. This is preferable to allowing the needle to deflect, which may result in the tip being several millimetres away from the intended site. 
15. Inject $4 \mu \mathrm{l}$ in 4 minutes, then wait another 4 minutes. Remove the syringe slowly, and stop any bleeding.

16. Remove the retractor and close the skin with 5-0 suture.

17. Transfer the rat to a warm chamber for recovery before returning it to the cage.

The surgery for CCP lesions involves more risk than for spinal cord lesions (e.g. haemorrhage) and post-operative complications are also more common. For the first 24 hours after the procedure, the rat should be housed individually and provided with a liquid/gel diet ad libitum and a long spout water bottle. If showing signs of pain, another dose of analgaesic can be administered several hours after surgery. Rats are often lethargic following the surgery but should regain their usual level of activity over 2-3 days. If a mild head tilt develops postoperatively, this should be monitored, with special feeding arrangements as required, and will usually resolve. In occasional cases, a rat may develop spontaneous rolling and will need to be culled.

\subsection{PROTOCOL: Dietary administration of cuprizone to mice}

The effects of cuprizone can depend on the species, strain, age and gender of the animals used. The literature standard is to use 8-week old male C57BL/6 mice, and feed a diet of $0.2 \%$ cuprizone for 4-6 weeks [52].

1. For a period of 4-6 weeks, substitute the regular feed with a $0.2 \%$ cuprizone diet. This should be replaced every 2-3 days, due to degradation of the toxin. Food intake can be estimated as $4-5 \mathrm{~g}$ per mouse per day.

2. Cuprizone poses a safety risk through skin contact or inhalation. For this reason, mice should be housed in sealed cages and diet/bedding changes carried out in a biological safety cabinet with appropriate waste disposal.

3. Mouse weights should be monitored throughout the experiment. Weight loss often occurs during the first 1-2 weeks of administration, with subsequent weight gain.

4. Mice are often returned to their regular diet for 1-2 weeks for a period of remyelination without ongoing demyelination.

Under these conditions, substantial demyelination is achieved whilst minimising clinical severity, which is frequent at higher doses [46, 74]. However, some weight loss is common and other signs have been reported including reduced social interaction [75] and impaired performance on a balance-beam task [76]. 


\section{Notes}

\subsection{Identifying Remyelination}

Several methods can be used to visualise and quantify myelin, including immunohistochemistry for myelin protein, light microscopy dyes including luxol fast blue or lipophilic fluorophores such as FluroMyelin (ThermoFisher). Magnetic resonance imaging (MRI) can also be useful tool to map myelin across the entire brain. However, these techniques are unable to distinguish between the myelin produced during remyelination and that originating in development. Thus, it can be difficult to determine whether an intervention has truly enhanced remyelination, or if more of the original sheaths have survived.

At a higher resolution it becomes clear that the dimensions of the myelin relative to its axon differs between myelination and remyelination. During development, myelin sheath thickness increases with larger diameter axons. In remyelination, this relationship is not maintained and thus a given diameter axon will be myelinated by a sheath which appears thinner and with shorter internodes than developmentally generated myelin (Fig 5) [77]. To be able to visualise this, the ultrastructure of the tissue must be very well preserved, and this is best done by perfusion-fixing animals in gluteraldehyde before embedding regions of interest resin [78]. Myelin sheath thickness can then be measured using $1 \mu \mathrm{m}$ sections stained with toluidine blue (with practice), or using electron microscopy. The thinner sheaths are less clear in regions of the CNS with smaller-diameter axons such as the corpus callosum, which can be problematic for interpreting electron microscopy in the cuprizone model [74].

\subsection{Other outcome measures for toxin-based models}

Besides imaging myelin sheaths themselves a wide variety of techniques have been applied to toxin-induced lesions to study the cells and molecular cues involved in demyelination and remyelination. To preserve the CNS for immunohistochemistry, a perfusion-fixation method using $4 \%$ paraformaldehyde in PBS is recommended. This delivers fixative rapidly and uniformly to all tissues, and is thus preferable to immersion-fixation [79]. In situ hybridisation is a useful alternative to immunohistochemistry, particularly to visualise transcripts encoding secreted factors or myelin sheath proteins, which would be difficult to relate to a cell body.

Flow cytometry has been applied to dissected lesion tissue with some success in the cuprizone model, although as the effected regions are relatively small this requires pooling of large numbers of mice [62]. In the lysolecithin model, inflammatory cells from the lesion and normal tissue have proved difficult to separate by flow cytometry, probably due to the small volume of affected tissue [80] relative to the more global inflammation which makes this possible in EAE [81]. In contrast to live cells, RNA and protein from a small region of CNS containing a lysolecithin lesion have been reliably used to investigate remyelination $[82,83]$. Total RNA has been successfully extracted from EB lesions very selectively using laser capture microdissection of frozen sections [35], revealing general patterns in gene expression at different timepoints during 
remyelination.

\subsection{Conclusions}

Toxin-based models can offer a valuable insight into the mechanisms of myelin diseases, and into broader aspects of regenerative medicine [84]. Predictable kinetics and a temporal dissociation between demyelination and remyelination are advantages of these paradigms that complement the findings of other model systems.

\subsection{Acknowledgements}

Thanks to Natalia Murphy for consultation regarding the ethidium bromide protocol, and to Joseph Guy for performing the cuprizone brain MRI. 


\section{References}

1. Morell P (1984) Myelin. doi: 10.1007/978-1-4757-1830-0

2. Blakemore WF (1975) Remyelination by Schwann cells of axons demyelinated by intraspinal injection of 6-aminonicotinamide in the rat. J Neurocytol 4:745-57. doi: 10.1007/BF01181634

3. Carroll WM, Jennings AR, Mastaglia FL (1984) Experimental demyelinating optic neuropathy induced by intra-neural injection of galactocerebroside antiserum. J Neurol Sci 65:125-135. doi: 10.1016/0022-510X(84)90077-7

4. Felts PA, Woolston AM, Fernando HB, et al (2005) Inflammation and primary demyelination induced by the intraspinal injection of lipopolysaccharide. Brain 128:1649-1666. doi: 10.1093/brain/awh516

5. Bakker DA, Ludwin SK (1987) Blood-brain barrier permeability during Cuprizone-induced demyelination. Implications for the pathogenesis of immune-mediated demyelinating diseases. J Neurol Sci 78:125-137. doi: 10.1016/0022-510X(87)90055-4

6. Torkildsen O, Brunborg LA, Myhr K-M, Bø L (2008) The cuprizone model for demyelination. Acta Neurol Scand Suppl 188:72-6. doi:

10.1111/j.1600-0404.2008.01036.x

7. Nishimoto S, Tanaka H, Okamoto M, et al (2015) Methylcobalamin promotes the differentiation of Schwann cells and remyelination in lysophosphatidylcholine-induced demyelination of the rat sciatic nerve. Front Cell Neurosci 9:298. doi: 10.3389/fncel.2015.00298

8. Zhang F, Yao SY, Whetsell WO, Sriram S (2013) Astrogliopathy and oligodendrogliopathy are early events in CNS demyelination. Glia 61:1261-1273. doi: 10.1002/glia.22513

9. Lauber K, Blumenthal SG, Waibel M, Wesselborg S (2004) Clearance of apoptotic cells: Getting rid of the corpses. Mol Cell 14:277-287. doi: 10.1016/S1097-2765(04)00237-0

10. Hall SM (1972) The effect of injections of lysophosphatidyl choline into white matter of the adult mouse spinal cord. J Cell Sci 10:535-546.

11. Blakemore WF (1976) Invasion of Schwann cells into the spinal cord of the rat following local injections of lysolecithin. Neuropathol Appl Neurobiol 2:21-39. doi: 10.1111/j.1365-2990.1976.tb00559.x

12. Blakemore WF (1978) Observations on remyelination in the rabbit spinal cord following demyelination induced by lysolecithin. Neuropathol Appl Neurobiol 4:47-59. doi: 10.1111/j.1365-2990.1978.tb00528.x

13. Nait-Oumesmar B, Decker L, Lachapelle F, et al (1999) Progenitor cells of the adult mouse subventricular zone proliferate, migrate and differentiate into oligodendrocytes after demyelination. Eur J Neurosci 11:4357-4366. doi: 10.1046/j.1460-9568.1999.00873.x

14. Woodruff RH, Franklin RJM (1999) Demyelination and remyelination of the caudal cerebellar peduncle of adult rats following stereotaxic injections of lysolecithin, ethidium bromide, and complement/antigalactocerebroside: A comparative study. Glia 25:216-228. doi: 10.1002/(SICI)1098-1136(19990201)25:3<216::AID-GLIA2>3.0.C0;2-L

15. Arnett HA, Fancy SPJ, Alberta JA, et al (2004) bHLH transcription factor Olig1 is required to repair demyelinated lesions in the CNS. Science 306:2111-5. doi: 10.1126/science.1103709

16. Jeffery ND, Blakemore WF (1995) Remyelination of mouse spinal cord 
axons demyelinated by local injection of lysolecithin. J Neurocytol 24:775781. doi: 10.1007/BF01191213

17. Gensert JM, Goldman JE (1997) Endogenous progenitors remyelinate demyelinated axons in the adult CNS. Neuron 19:197-203. doi: 10.1016/S0896-6273(00)80359-1

18. Gilson J, Blakemore WF (1993) Failure of remyelination in areas of demyelination produced in the spinal cord of old rats. Neuropathol Appl Neurobiol 19:173-181.

19. Weltzien HU (1979) Cytolytic and membrane-perturbing properties of lysophosphatidylcholine. BBA - Rev Biomembr 559:259-287. doi: 10.1016/0304-4157(79)90004-2

20. Gregson NA, Hall SM (1973) A quantitative analysis of the effects of the intraneural injection of lysophosphatidyl choline. J Cell Sci 13:257-77.

21. Quinn MT, Parthasarathy S, Steinberg D (1988) Lysophosphatidylcholine: a chemotactic factor for human monocytes and its potential role in atherogenesis. Proc Natl Acad Sci U S A 85:2805-9.

22. McMurray HF, Parthasarathy S, Steinberg D (1993) Oxidatively Modified Low-Density-Lipoprotein Is a Chemoattractant for Human T-Lymphocytes. J Clin Invest 92:1004-1008. doi: 10.1172/JCI116605

23. Ngwenya BZ, Yamamoto N (1985) Activation of peritoneal macrophages by lysophosphatidylcholine. 839:9-15.

24. Huang YH, Schäfer-Elinder L, Wu R, et al (1999) Lysophosphatidylcholine (LPC) induces proinflammatory cytokines by a platelet-activating factor (PAF) receptor-dependent mechanism. Clin Exp Immunol 116:326-31. doi: 10.1046/j.1365-2249.1999.00871.x

25. Schilling T, Lehmann F, Rückert B, Eder C (2004) Physiological mechanisms of lysophosphatidylcholine-induced de-ramification of murine microglia. J Physiol 557:105-120. doi:

10.1113/jphysiol.2004.060632

26. Ousman SS, David S (2001) MIP-1alpha, MCP-1, GM-CSF, and TNF-alpha control the immune cell response that mediates rapid phagocytosis of myelin from the adult mouse spinal cord. J Neurosci 21:4649-56.

27. Ghasemlou N, Jeong SY, Lacroix S, David S (2007) T cells contribute to lysophosphatidylcholine-induced macrophage activation and demyelination in the CNS. Glia 55:294-302. doi: 10.1002/glia.20449

28. Kotter MR, Setzu A, Sim FJ, et al (2001) Macrophage depletion impairs oligodendrocyte remyelination following lysolecithin-induced demyelination. Glia 35:204-12. doi: 10.1002/glia.1085

29. Bieber AJ, Kerr S, Rodriguez M (2003) Efficient central nervous system remyelination requires T cells. Ann Neurol 53:680-684. doi: 10.1002/ana.10578

30. Miron V, Franklin R (2014) Macrophages and CNS remyelination. J Neurochem 1-7. doi: 10.1111/jnc.12705

31. Liu L, Belkadi A, Darnall L, et al (2010) CXCR2-positive neutrophils are essential for cuprizone-induced demyelination: relevance to multiple sclerosis. Nat Neurosci 13:319-326. doi: 10.1038/nn.2491

32. Birgbauer E, Rao TS, Webb M (2004) Lysolecithin induces demyelination in vitro in a cerebellar slice culture system. J Neurosci Res 78:157-166. doi: 10.1002/jnr.20248 
33. Zhang H, Jarjour AA, Boyd A, Williams A (2011) Central nervous system remyelination in culture - A tool for multiple sclerosis research. Exp Neurol 230:138-148. doi: 10.1016/j.expneurol.2011.04.009

34. Miron VE, Ludwin SK, Darlington PJ, et al (2010) Fingolimod (FTY720) enhances remyelination following demyelination of organotypic cerebellar slices. Am J Pathol 176:2682-2694. doi: 10.2353/ajpath.2010.091234

35. Huang JK, Jarjour AA, Nait Oumesmar B, et al (2011) Retinoid X receptor gamma signaling accelerates CNS remyelination. Nat Neurosci 14:45-53. doi: $10.1038 / \mathrm{nn} .2702$

36. Waring MJ (1965) Complex Formation between Ethidium Bromide and Nucleic Acids. J Mol Biol 13:269-282. doi: 10.1016/S00222836(65)80096-1

37. Chowdhury AR, Bakshi R, Wang J, et al (2010) The killing of African trypanosomes by ethidium bromide. PLoS Pathog. doi:

10.1371/journal.ppat.1001226

38. Blakemore WF (1982) Ethidium bromide induced demyelination in the spinal cord of the cat. Neuropathol Appl Neurobiol 8:365-375.

39. Goudarzvand M, Choopani S, Shams A, et al (2016) Focal Injection of Ethidium Bromide as a Simple Model to Study Cognitive Deficit and Its Improvement. Basic Clin Neurosci J 7:63-73.

40. Sim FJ, Zhao C, Penderis J, Franklin RJM (2002) The age-related decrease in CNS remyelination efficiency is attributable to an impairment of both oligodendrocyte progenitor recruitment and differentiation. J Neurosci 22:2451-2459. doi: 10.1097/00041327-200306000-00025

41. Zawadzka M, Rivers LE, Fancy SPJ, et al (2010) CNS-resident glial progenitor/stem cells produce Schwann cells as well as oligodendrocytes during repair of CNS demyelination. Cell Stem Cell 6:578-90. doi: 10.1016/j.stem.2010.04.002

42. Monteiro De Castro G, Deja NA, Ma D, et al (2015) Astrocyte Activation via Stat3 Signaling Determines the Balance of Oligodendrocyte versus Schwann Cell Remyelination. Am J Pathol 185:2431-2440. doi: 10.1016/j.ajpath.2015.05.011

43. Graça DL, Blakemore WF (1986) Delayed remyelination in rat spinal cord following ethidium bromide injection. Neuropathol Appl Neurobiol 12:593-605.

44. Kotter MR, Li W-W, Zhao C, Franklin RJM (2006) Myelin impairs CNS remyelination by inhibiting oligodendrocyte precursor cell differentiation. J Neurosci 26:328-32. doi: 10.1523/JNEUROSCI.2615-05.2006

45. Carlton WW (1966) Response of mice to the chelating agents sodium diethyldithiocarbamate, alpha-benzoinoxime, and biscyclohexanone oxaldihydrazone. Toxicol Appl Pharmacol 8:512-21. doi: 10.1016/0041008X(66)90062-7

46. Hiremath MM, Saito Y, Knapp GW, et al (1998) Microglial/macrophage accumulation during cuprizone-induced demyelination in C57BL/6 mice. J Neuroimmunol 92:38-49. doi: 10.1016/S0165-5728(98)00168-4

47. Steelman AJ, Thompson JP, Li J (2012) Demyelination and remyelination in anatomically distinct regions of the corpus callosum following cuprizone intoxication. Neurosci Res 72:32-42. doi: 10.1016/j.neures.2011.10.002

48. Mason JL, Jones JJ, Taniike M, et al (2000) Mature oligodendrocyte 
apoptosis precedes IGF-1 production and oligodendrocyte progenitor accumulation and differentiation during demyelination/remyelination. J Neurosci Res 61:251-262. doi: 10.1002/1097-

4547(20000801)61:3<251::AID-JNR3>3.0.CO;2-W

49. Morell P, Barrett C V, Mason JL, et al (1998) Gene expression in brain during cuprizone-induced demyelination and remyelination. Mol Cell Neurosci 12:220-7. doi: 10.1006/mcne.1998.0715

50. Zoupi L, Markoullis K, Kleopa KA, Karagogeos D (2013) Alterations of juxtaparanodal domains in two rodent models of CNS demyelination. Glia 61:1236-1249. doi: 10.1002/glia.22511

51. Manrique-Hoyos N, Jürgens T, Grønborg M, et al (2012) Late motor decline after accomplished remyelination: impact for progressive multiple sclerosis. Ann Neurol 71:227-44. doi: 10.1002/ana.22681

52. Praet J, Guglielmetti C, Berneman Z, et al (2014) Cellular and molecular neuropathology of the cuprizone mouse model: Clinical relevance for multiple sclerosis. Neurosci Biobehav Rev 47:485-505. doi: 10.1016/j.neubiorev.2014.10.004

53. Venturini G (1973) Enzymic activities and sodium, potassium and copper concentrations in mouse brain and liver after cuprizone treatment in vivo. J Neurochem 21:1147-1151. doi: 10.1111/j.1471-4159.1973.tb07569.x

54. Benetti F, Ventura M, Salmini B, et al (2010) Cuprizone neurotoxicity, copper deficiency and neurodegeneration. Neurotoxicology 31:509-517. doi: 10.1016/j.neuro.2010.05.008

55. Zatta P, Raso M, Zambenedetti P, et al (2005) Copper and zinc dismetabolism in the mouse brain upon chronic cuprizone treatment. Cell Mol Life Sci 62:1502-1513. doi: 10.1007/s00018-005-5073-8

56. Bénardais K, Kotsiari A, Škuljec J, et al (2013) Cuprizone [bis(cyclohexylidenehydrazide)] is selectively toxic for mature oligodendrocytes. Neurotox Res 24:244-250. doi: 10.1007/s12640-0139380-9

57. Bradl M, Lassmann H (2010) Oligodendrocytes: Biology and pathology. Acta Neuropathol 119:37-53. doi: 10.1007/s00401-009-0601-5

58. Suzuki K (1969) Giant hepatic mitochondria: production in mice fed with cuprizone. Science 163:81-2. doi: 10.1126/science.163.3862.81

59. Doan V, Kleindienst AM, Mcmahon EJ, et al (2013) Abbreviated exposure to cuprizone is sufficient to induce demyelination and oligodendrocyte loss. J Neurosci Res 91:363-373. doi: 10.1002/jnr.23174

60. Clarner T, Janssen K, Nellessen L, et al (2015) CXCL10 Triggers Early Microglial Activation in the Cuprizone Model. J Immunol 194:3400-3413. doi: 10.4049/jimmunol.1401459

61. Arnett HA, Mason J, Marino M, et al (2001) TNF alpha promotes proliferation of oligodendrocyte progenitors and remyelination. Nat Neurosci 4:1116-22. doi: 10.1038/nn738

62. Voß EV, Škuljec J, Gudi V, et al (2012) Characterisation of microglia during de- and remyelination: Can they create a repair promoting environment? Neurobiol Dis 45:519-528. doi: 10.1016/j.nbd.2011.09.008

63. Lampron A, Larochelle A, Laflamme N, et al (2015) Inefficient clearance of myelin debris by microglia impairs remyelinating processes. J Exp Med 212:481-95. doi: 10.1084/jem.20141656 
64. Mason JL, Langaman C, Morell P, et al (2001) Episodic demyelination and subsequent remyelination within the murine central nervous system: Changes in axonal calibre. Neuropathol Appl Neurobiol 27:50-58. doi: 10.1046/j.0305-1846.2001.00301.x

65. Lindner M, Fokuhl J, Linsmeier F, et al (2009) Chronic toxic demyelination in the central nervous system leads to axonal damage despite remyelination. Neurosci Lett 453:120-125. doi: 10.1016/j.neulet.2009.02.004

66. Sachs HH, Bercury KK, Popescu DC, et al (2014) A New Model of Cuprizone-Mediated Demyelination/Remyelination. ASN Neuro 6:1-16. doi: 10.1177/1759091414551955

67. Olitsky PK, Yager RH (1949) Experimental disseminated encephalomyelitis in white mice. J Exp Med 90:213-224. doi: 10.1084/jem.90.3.213

68. Robinson AP, Harp CT, Noronha A, Miller SD (2014) The experimental autoimmune encephalomyelitis (EAE) model of MS: utility for understanding disease pathophysiology and treatment. 173-189. doi: 10.1016/B978-0-444-52001-2.00008-X.The

69. Dal Canto MC, Lipton HL (1975) Primary demyelination in Theiler's virus infection. An ultrastructural study. Lab Invest 33:626-37.

70. Mecha M, Carrillo-Salinas FJ, Mestre L, et al (2013) Viral models of multiple sclerosis: Neurodegeneration and demyelination in mice infected with Theiler's virus. Prog Neurobiol 101-102:46-64. doi: 10.1016/j.pneurobio.2012.11.003

71. Griffiths IR, Schneider A, Anderson J, Nave KA (1995) Transgenic and natural mouse models of proteolipid protein (PLP)-related dysmyelination and demyelination. Brain Pathol 5:275-281.

72. Wolfensohn S, Hawkins P, Lilley E, et al (2013) Reducing suffering in experimental autoimmune encephalomyelitis (EAE). J Pharmacol Toxicol Methods 67:169-176. doi: 10.1016/j.vascn.2013.01.009

73. Paxinos G, Watson C (2013) The Rat Brain in Stereotaxic Coordinates: Hard Cover Edition. Elsevier Science

74. Stidworthy MF, Genoud S, Suter U, et al (2003) Quantifying the early stages of remyelination following cuprizone-induced demyelination. Brain Pathol 13:329-339. doi: 10.1111/j.1750-3639.2003.tb00032.x

75. Xu H, Yang H, Clough R, Browning R (2009) Behavioral and Neurobiological Changes in C57BL / 6 Mice Exposed to Cuprizone. 123:418-429. doi: 10.1037/a0014477

76. Hagemeyer N, Boretius S, Ott C, et al (2012) Erythropoietin attenuates neurological and histological consequences of toxic demyelination in mice. Mol Med 18:628-35. doi: 10.2119/molmed.2011.00457

77. Franklin RJM, Zhao C, Lubetzki C, Ffrench-Constant C (2013) Endogenous Remyelination in the CNS. In: Myelin Repair Neuroprotection Mult. Scler. Springer US, Boston, MA, pp 71-92

78. Blakemore WF, Franklin RJM (2008) Remyelination in experimental models of toxin-induced demyelination. Curr Top Microbiol Immunol 318:193-212. doi: 10.1007/978-3-540-73677-6-8

79. Gage GJ, Kipke DR, Shain W (2012) Whole animal perfusion fixation for rodents. J Vis Exp e3564. doi: 10.3791/3564 
80. Döring A, Sloka S, Lau L, et al (2015) Stimulation of monocytes, macrophages, and microglia by amphotericin $\mathrm{B}$ and macrophage colonystimulating factor promotes remyelination. J Neurosci 35:1136-48. doi: 10.1523/JNEUROSCI.1797-14.2015

81. Goncalves DaSilva A, Yong VW (2009) Matrix metalloproteinase-12 deficiency worsens relapsing-remitting experimental autoimmune encephalomyelitis in association with cytokine and chemokine dysregulation. Am J Pathol 174:898-909. doi: 10.2353/ajpath.2009.080952

82. Zhao C, Li W-W, Franklin RJM (2006) Differences in the early inflammatory responses to toxin-induced demyelination are associated with the agerelated decline in CNS remyelination. Neurobiol Aging 27:1298-307. doi: 10.1016/j.neurobiolaging.2005.06.008

83. Boyd A, Zhang H, Williams A (2013) Insufficient OPC migration into demyelinated lesions is a cause of poor remyelination in MS and mouse models. Acta Neuropathol 125:841-859. doi: 10.1007/s00401-013-1112-y

84. Aurora AB, Olson EN (2014) Immune modulation of stem cells and regeneration. Cell Stem Cell 15:14-25. doi: 10.1016/j.stem.2014.06.009 


\section{Figures}

Figure 1: Schematic overview of the mechanisms by which lysolecithin (a), ethidium bromide (b) and cuprizone (c) cause demyelination.

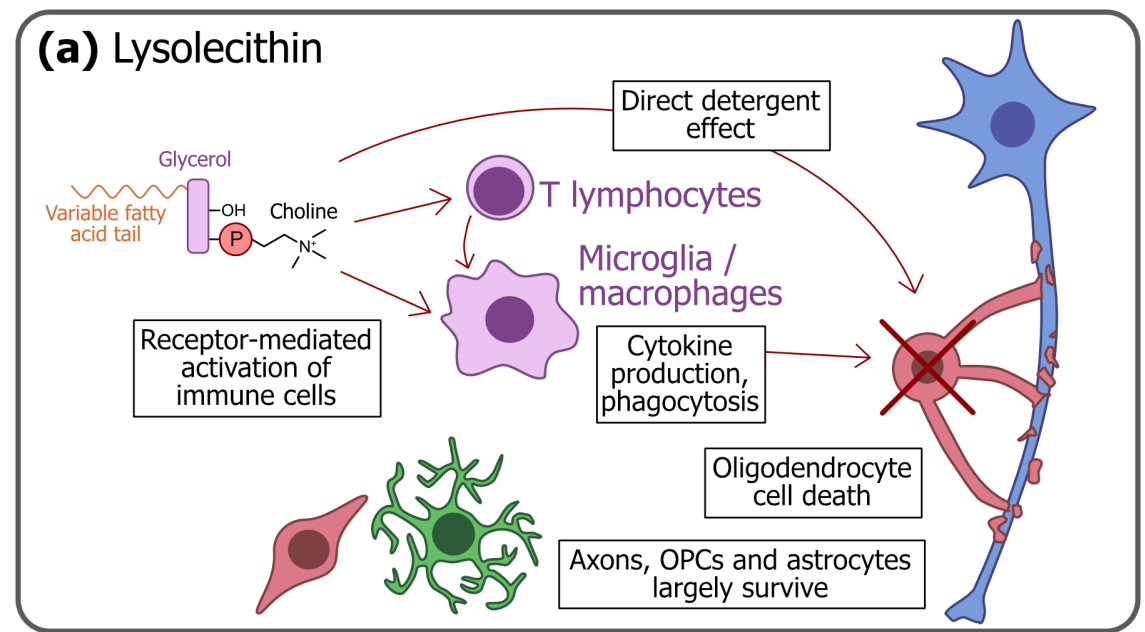

\section{(b) Ethidium Bromide}
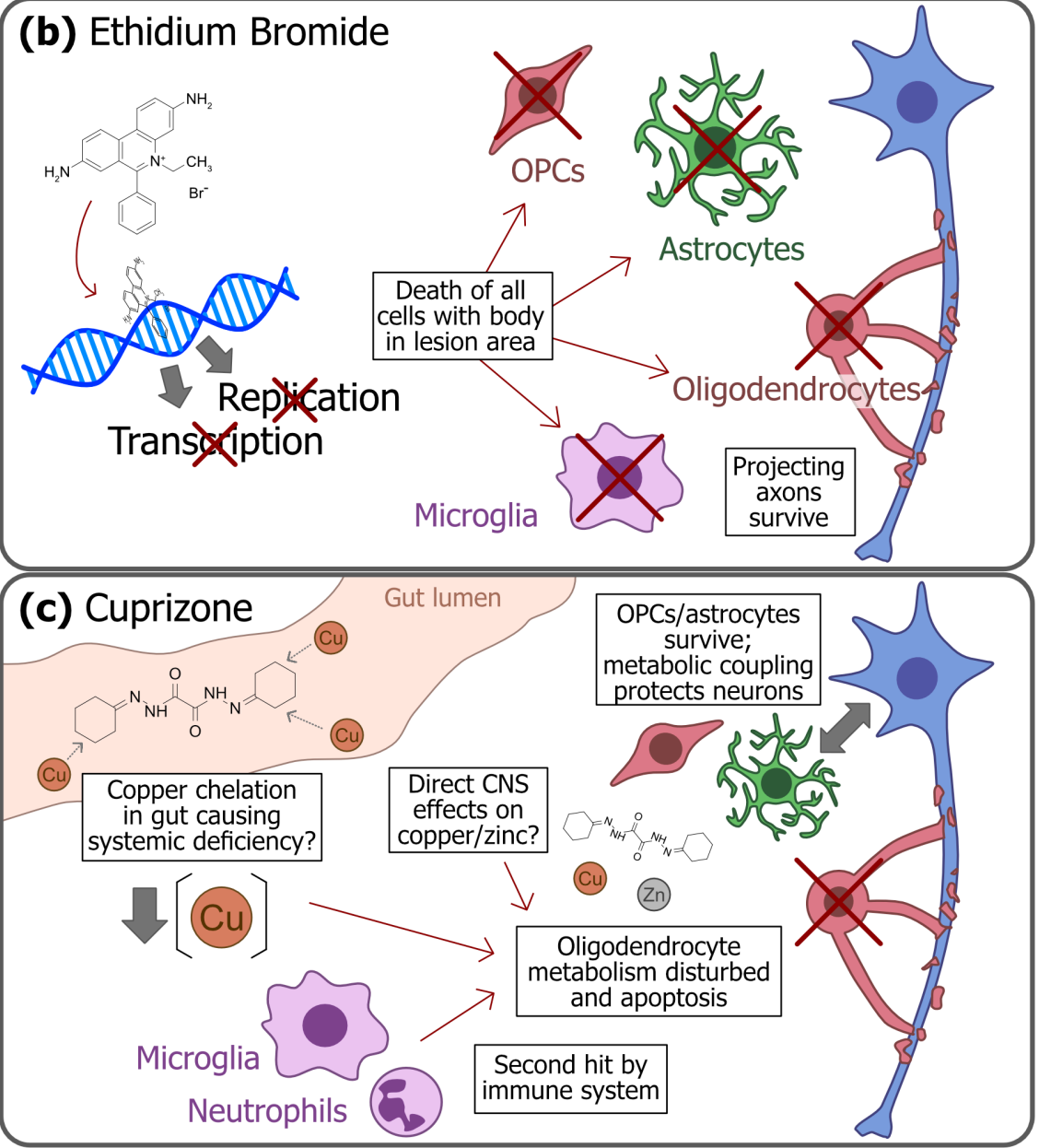
Figure 2: T2*-weighted gadolinium-enhanced Magnetic Resonance Image (MRI) of brain hemispheres from a mouse fed a $0.2 \%$ cuprizone diet for 5 weeks (right) and a control animal (left). Myelin appears dark. The cuprizone-treated mouse shows marked demyelination in the corpus callosum (white boxes), seen here at its caudal end.

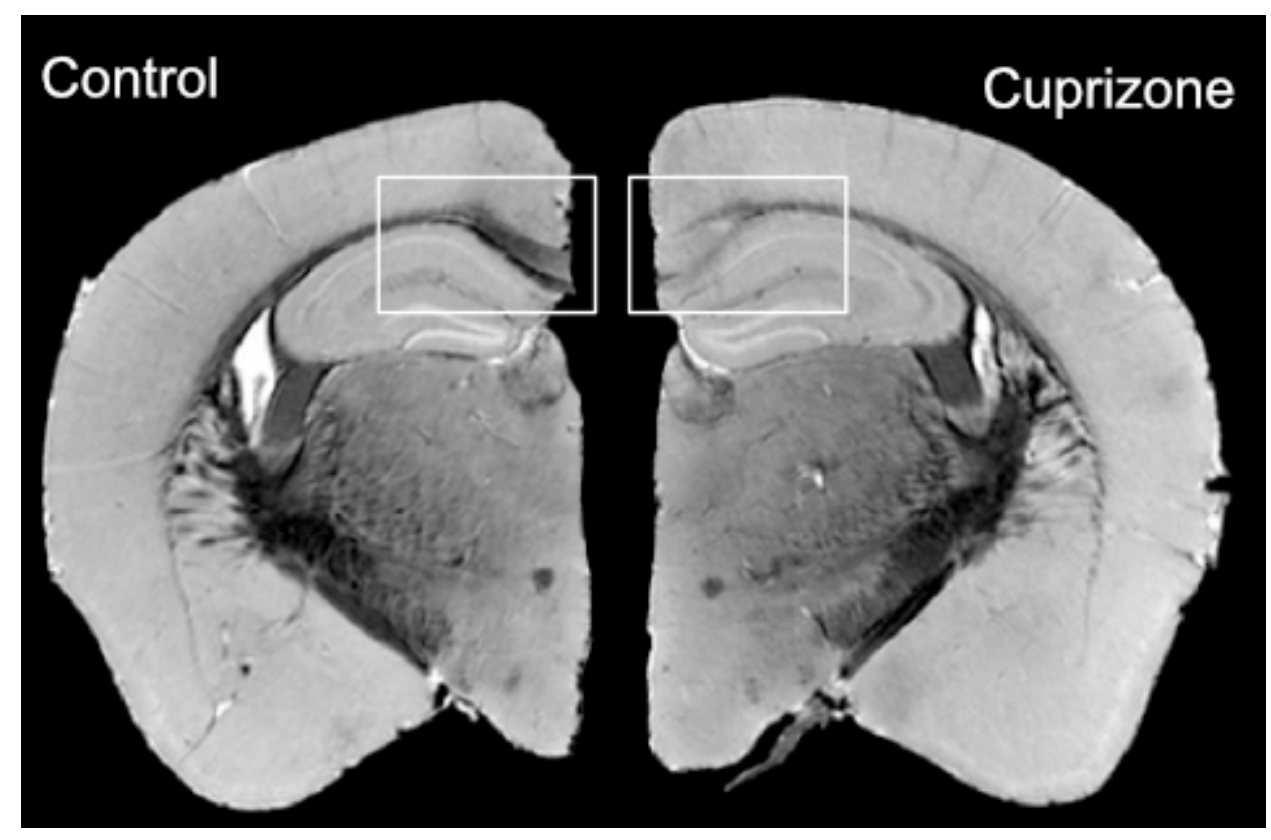

Figure 3: Injection of lysolecithin into the ventral white matter of the spinal cord.

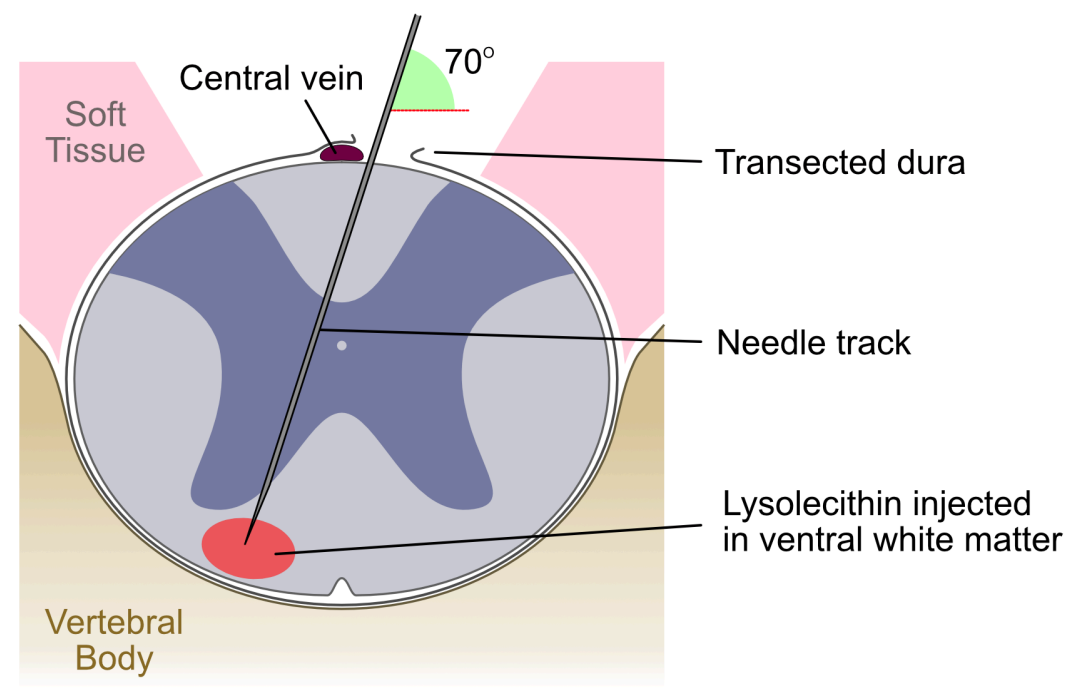


Figure 4: The dorsal surface of the rat skull. The lamba, $\lambda$, is defined as the midpoint of the curve of best fit along the lambdoid suture, rather than the intersection between the lambdoid and sagittal sutures.

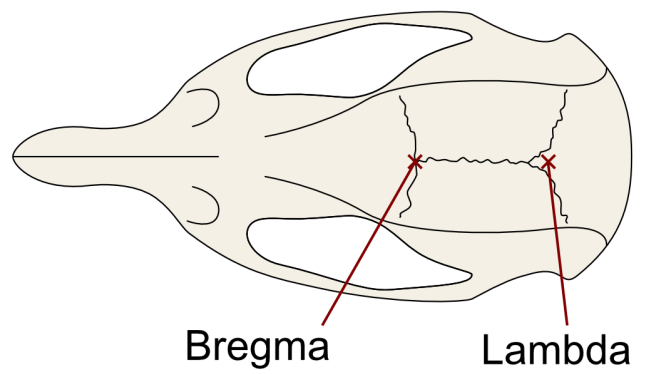

Figure 5: Electron micrographs of unlesioned white matter, demyelination and remyelination, highlighting the thinner appearance of the remyelinated sheaths Unlesioned white matter Demyelination Remyelination
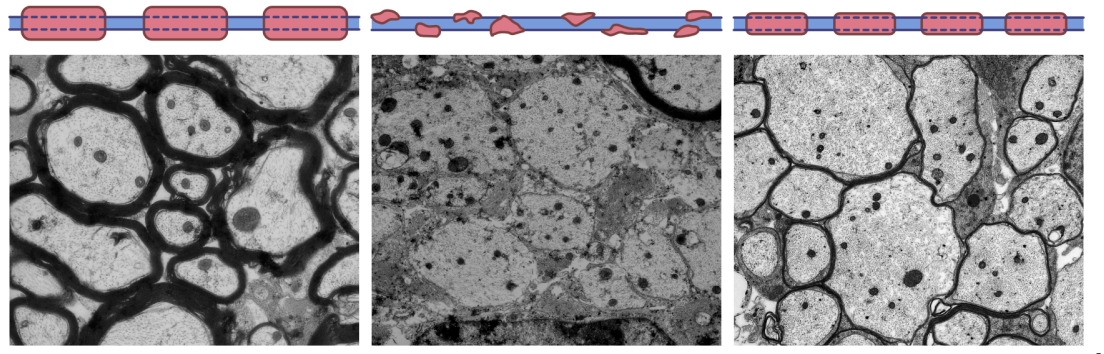